\title{
Towards the Electron Spectroscopy Graphene Fingerprint
}

\author{
Lester Lampert, Andrew Barnum, and Jun Jiao
}

Department of Mechanical \& Materials Engineering and Department of Physics, Portland State University, Portland, OR, USA.

Graphene research continues to demonstrate that this single atom-thick layer of carbon atom material has unprecedented properties for various applications within electronic and optical devices. Observed phenomena from graphene-based devices are largely dependent upon coupling of graphene with the underlying substrate. Especially, the number of layers of graphene, the defect density, and dopants may affect the performance of the devices. To date, Raman spectroscopy has been a dominant tool for graphene property characterizations by establishing fingerprint spectra to determine relative doping levels, number of layers, stacking symmetry, temperature, etc. [1]. Recently, Auger electron spectroscopy (AES) has been slowly gaining popularity for graphene characterization due to its intrinsic surface sensitivity and its ability to measure electron interactions with the surrounding environment. Although there is a wealth of AES data available for graphite and highly ordered pyrolytic graphite (HOPG) [2], including some work with carbon nanotubes [3], as well as some recent work to determine the number of graphene layers by utilizing a calculated electron inelastic mean free path [4], there has yet to be a satisfying description of some AES spectra phenomena observed with graphene. Here, we report AES, X-ray photoelectron spectroscopy (XPS) and Raman spectroscopy of CVD graphene, amorphous carbon, and CVD diamond towards the development of an AES fingerprint spectrum of graphene on various substrates.

Amorphous carbon $(\mathrm{a}-\mathrm{C})$ films were deposited with a carbon rod coater (target thickness $=4 \mathrm{~nm}$ ) onto $\mathrm{Si} / \mathrm{SiO}_{2}$ wafers. CVD diamond samples (diamond on oxide - DOI) were purchased from MTI Corporation ( $2 \mu \mathrm{m}$ thick). CVD graphene was grown with a vertical cold wall furnace held under vacuum at $750^{\circ} \mathrm{C}$ during the annealing/growth phase with a mixture of $\mathrm{H}_{2}, \mathrm{CH}_{4}$, and Ar gases. AES and XPS data was acquired with a PHI Versaprobe II AES/XPS/UPS microprobe. Raman spectra were collected with a Horiba HR800 Raman spectrometer microprobe (ex. = $532 \mathrm{~nm}$ ).

AES survey and C KVV spectra as depicted in Fig. 1 were acquired for different carbon allotropes: amorphous carbon (a-C), CVD graphene ( $\mathrm{sp}^{2}$ ) supported by $\mathrm{SiO}_{2}$, and $\mathrm{CVD}$ diamond $\left(\mathrm{sp}^{2} / \mathrm{sp}^{3}\right)$. From Fig. $1 \mathrm{~b}$, it can be seen that the $\mathrm{D}$ parameter for each spectra is $22.8,23.1$, and $21.8 \mathrm{eV}$ (bottom to top). While the D parameter is similar for a-C and CVD graphene on $\mathrm{SiO}_{2}$, the shoulder at $265 \mathrm{eV}$ is suppressed for a-C. This is in good agreement with previously published results to denote the difference between amorphous carbon and a graphitic-like AES spectra [3]. Also, at $\sim 255 \mathrm{eV}$ and $\sim 260 \mathrm{eV}$ there exists a prominent peak for the CVD diamond spectra, which may indicate the contributions from $\mathrm{sp}^{3}$ carbon. Fig. 2 demonstrates the Raman and XPS spectra of the samples investigated in Fig. 1. From the Raman spectra, all allotropes share a peak $\sim 1590 \mathrm{~cm}^{-1}$ ( $\mathrm{G}$ band), which is indicative of each sample having some $\mathrm{sp}^{2}$ carbon and is reflected in the presence of peaks $\sim 252 \mathrm{eV}$ in Fig. 1b. AES of graphene on different substrates is depicted in Fig. 3. In Fig. 3a, it can be seen the CVD graphene on $\mathrm{SiO}_{2}$ has a noticeable peak at $240 \mathrm{eV}$ attributed to a two-hole plasmon replica effect [5]. D parameters for Fig. 3b are 23.1, 21.8, and 22.8 (bottom to top). The $\mathrm{D}$ parameter of graphene/Cu is more diamond-like and that of graphene/Ni is more a-C-like, most likely due to graphite-like structure. Coupled with established techniques such as XPS and Raman, new properties of graphene can be elucidated from an understanding of the AES spectra of graphene within different environments. 
References:

[1] A. C. Ferrari et al, Nature Nanotechnology 8 (2013), p. 235-246.

[2] H. Hashimoto et al. Surf. Interface Anal. 35 (2003), p. 19-23.

[3] K. B. K. Teo et al, J. Vac. Sci. Technol. B 20 (2002), p. 116-121.

[4] M. Xu et al, ACS Nano 4 (2010), p. 2937-2945.

[5] E. Perfetto et al, Physical Review B 76 (2007), p. 233408 (1-4).

[6] This study is supported in part by Oregon Metals Initiative and NSF awards No. 1229663 and No. 1240121.
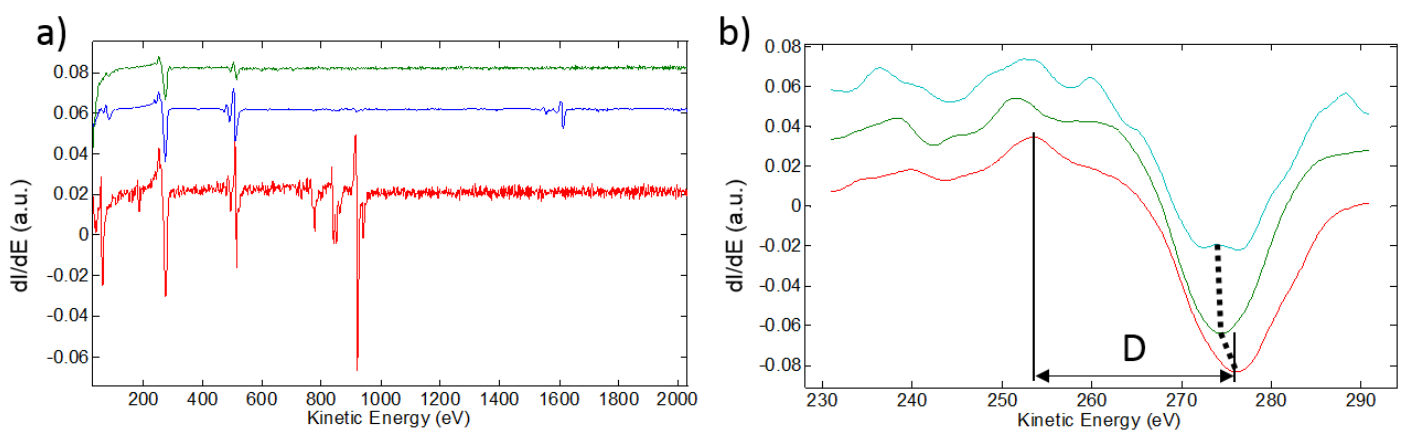

Figure 1. (a) Differentiated AES survey spectra of a-C film (bottom, red line), CVD graphene on $\mathrm{SiO}_{2}$ (middle, blue line), and CVD diamond (top, green line); (b) C KVV differentiated AES spectra of a-C film (bottom, red line), CVD graphene on $\mathrm{SiO}_{2}$ (middle, green line), and CVD diamond (top, cyan line).
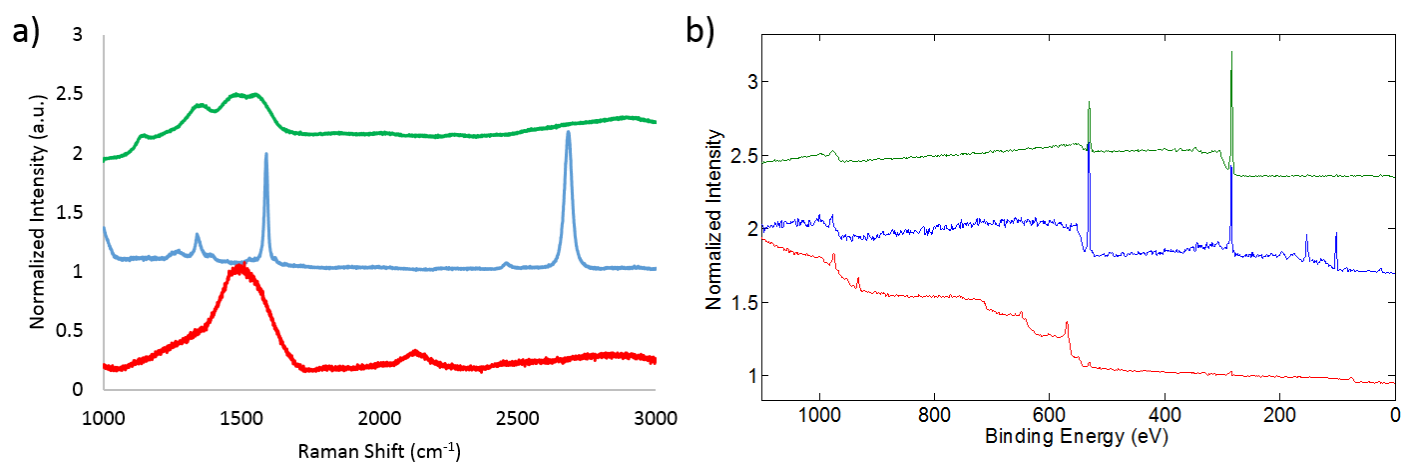

Figure 2. (a) Raman spectra and (b) XPS survey spectra of a-C film (bottom, red line), CVD graphene on $\mathrm{SiO}_{2}$ (middle, blue line), and CVD diamond (top, green line).
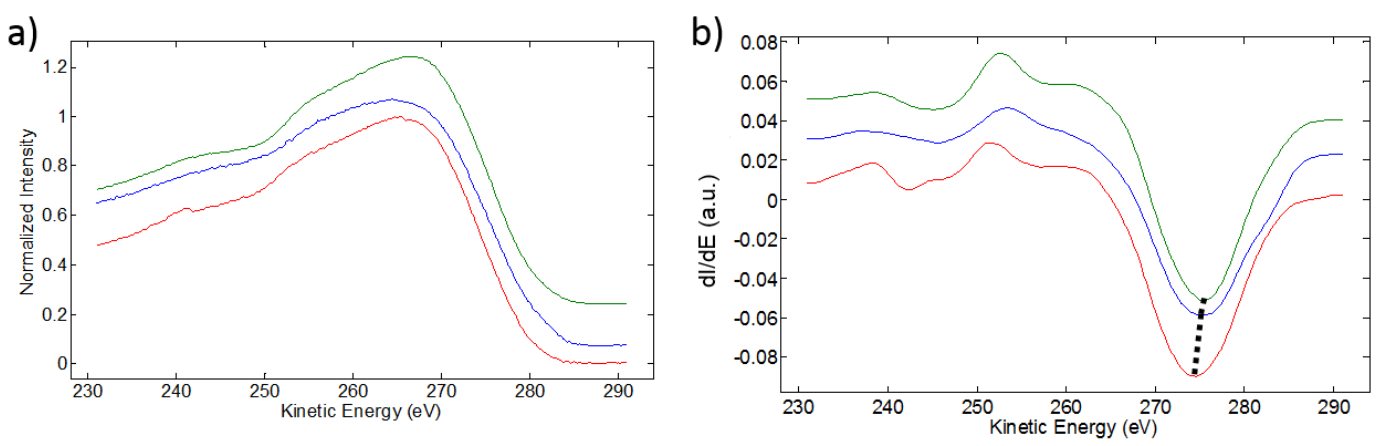

Figure 3. (a) Non-differentiated and (b) differentiated AES C KVV spectra for CVD graphene on $\mathrm{SiO}_{2}$ (bottom, red line), $\mathrm{Cu}$ (middle, blue line), and $\mathrm{Ni}$ (top, green line). 